\title{
FLAVONÓIDE E TRITERPENOS DE Stigmaphyllom paralias
}

\author{
Jorge M. David* e Fátima A. Santos \\ Instituto de Química, Universidade Federal da Bahia, 40170-290 Salvador - BA \\ Maria Lenise da S. Guedes \\ Instituto de Biologia, Universidade Federal da Bahia, 40170-290 Salvador - BA \\ Juceni P. David \\ Faculdade de Farmácia, Universidade Federal da Bahia, 40170-290 Salvador - BA
}

Recebido em 24/6/02; aceito em 13/12/02

\begin{abstract}
FLAVONOID AND TRITERPENES FROM STIGMAPHYLLOM PARALIAS. Stigmaphyllom paralias is a herb belonging to the family Malpighiaceae that occurs in sand soil of brazilian " restinga". This is the first report regarding phytochemical study with this species. The hexane extract of the aerial parts of plant afforded the triterpenes friedelin, lupenone, 3-oxo- $\alpha$-amirin and 3-oxo$\beta$-amirin, the mixture of $\alpha$-amirinyl palmitate and stearate, lupeol and 3,4-seco-friedelan-3-oic acid. The AcOEt extract yielded the flavonoid luteolin-7-rutinoside. All compounds were characterized by analysis of spectrometric data and the fatty acids esterified with a-amirine were identified by GC/MS of methyl derivatives of transesterified products. This is the first natural occurence of 3,4-seco-friedelan-3-oic acid and the ${ }^{13} \mathrm{C}$ NMR spectral data were inequivocally assigned by two-dimensional techniques. This work also permitted to correct the ${ }^{13} \mathrm{C}$ NMR resonances attributed to methyl groups $\mathrm{C}-26$ and $\mathrm{C}-27$ of fridelin.
\end{abstract}

Keywords: Stigmaphyllom paralias; 3,4-seco-friedelan-3-oic acid; luteolin-7-ruthenoside.

\section{INTRODUÇÃO}

A família Malpighiaceae é formada por aproximadamente 800 espécies especialmente americanas distribuídas em 60 gêneros, de ocorrência em regiões tropicais ${ }^{1}$. Os representantes mais importantes desta família são os cipós originários da Amazônia, do gênero Banisteropsis, que são empregados pelos indígenas como preparados alucinógenos em rituais. Os alcalóides do tipo $\beta$-carbolíneos são as substâncias responsáveis pela atividade alucinógena apresenta$\mathrm{da}^{2}$.

No nordeste brasileiro ocorrem diversas espécies dos gêneros Byrsonima, Camarea, Galphimia, Stigmaphyllom e Peixotoa. Apesar desse grande número de espécies vegetais, pouco é conhecido acerca da constituição química da família. Os poucos trabalhos na literatura reportam a presença de triterpenos em $B$. verbascifolia e $B$. microphylla ${ }^{3}$ e, flavonóides e esteróides em $B$. variabilis e $S$. tometosum $^{4}$. Além desta última espécie, outros estudos de abordagem fitoquímica do gênero Stigmaphyllom são restritos a espécies que ocorrem nas Guianas. Das raízes e folhas de $S$. sagitattum e raízes de $S$. convolvulifolium foram isolados iridóides ${ }^{5}$. A presença de iridóides na família Malpighiaceae é uma informação relevante pois, segundo a classificação de Dahlgreen, esta família da ordem Polygalales encontra-se distante das ordens que produzem esta classe de metabólito. No Brasil a maior parte das espécies deste gênero são conhecidas por serem utilizadas como alimentos, como por exemplo $S$. fulgens (cajuçára), além do grupo de espécies do gênero que são conhecidas como cipó guassú ${ }^{1}$. Deste modo, os estudos fitoquímicos com plantas desta família são justificados. Neste trabalho está descrito o isolamento das substâncias presentes nos extratos hexânico e acetato de etila das partes aéreas de $S$. paralias A. Juss., um arbusto normalmente baixo, de ocorrência comum nas restingas do litoral e dos tabuleiros da Bahia.

*e-mail: jmdavid@ufba.br

\section{PARTE EXPERIMENTAL}

Os espectros no UV foram registrados em espectrofotometro da Varian mod. DS500. Os espectros no Infravermelho foram registrados no espectrômetro Jasco mod. Valor III, enquanto que os espectros de RMN foram obtidos em espectrômetro Gemini 300 da Varian, sendo que o referencial interno utilizado foram os picos dos solventes deuterados em relação ao sinal do TMS. Os espectros HMBC e HMQC foram registrados em equipamento da GE Omega $500 \mathrm{MHz}$. Os espectros de massas foram obtidos por inserção direta em equipamento da Finnigan mod. MAT 90 e em CG acoplado a detetor de massas da Varian mod. Saturn II para análise dos ésteres metílicos.

As partes aéreas de espécimens de $S$. paralias foram coletadas nas dunas da restinga do Parque Metropolitano do Abaeté, Salvador (BA). Um exemplar encontra-se catalogado no Herbário Alexandre Leal Costa do Instituto de Biologia da UFBA sob número 54296.

Após secagem e trituração, $215 \mathrm{~g}$ da planta foram submetidas à maceração com metanol. O extrato metanólico ( $17 \mathrm{~g})$ foi particionado entre hexano: $\mathrm{MeOH}, \mathrm{CHCl}_{3} / \mathrm{MeOH}: \mathrm{H}_{2} \mathrm{O}$ e $\mathrm{AcOEt} / \mathrm{H}_{2} \mathrm{O}$ fornecendo as fases hexânica $(1,8 \mathrm{~g})$, a fase $\mathrm{CHCl}_{3}(3,2 \mathrm{~g})$ e AcOEt $(10,5 \mathrm{~g})$, respectivamente.

$\mathrm{O}$ extrato hexânico foi submetido à $\mathrm{CC}$ sob sílica gel, eluída com misturas de hexano:AcOEt com gradiente crescente de polaridade. Foram obtidas 45 frações de $50 \mathrm{ml}$ cada e, a análise das frações obtidas por CCD usando reagente de Lieberman-Buchard permitiu o reagrupamento daquelas cuja composição era semelhante. A fração eluída com hex:AcOEt (95:5) foi submetida a nova CC sob sílica gel eluída com hexano:AcOEt (95:5). Foram recolhidas 40 frações de $13 \mathrm{ml}$ cada e desta maneira isolados friedelina $(\mathbf{1}, 43 \mathrm{mg}), 98 \mathrm{mg}$ da mistura dos triterpenos lupenona, $\alpha$ - e $\beta$-amirinona, além da mistura de palmitato e estearato de $\alpha$-amirina $(\mathbf{2 a}+\mathbf{2 b}, 21 \mathrm{mg})$. Da fração eluída com hex:AcOEt (9:1) foi obtido o lupeol (24 mg). As frações eluídas com hex:AcOEt (8:2) foram agrupadas com base em CCD e submetidas à permeação com Sephadex LH-20 utilizando-se uma mistura de $\mathrm{MeOH}$ : $\mathrm{CH}_{2} \mathrm{Cl}_{2}$ (1:1). Este procedimento permitiu o isolamento do ácido 3,4-seco-friedelan-3-óico (3, $22 \mathrm{mg})$. 
$\mathrm{O}$ extrato AcOEt foi submetido à $\mathrm{CC}$ sob sílica gel 60 e eluído com misturas de $\mathrm{CHCl}_{3}: \mathrm{MeOH}$ nas proporções 95:5, 9:1 e 4:1, sendo coletadas frações de $50 \mathrm{ml}$ cada. As frações eluídas com a mistura de $\mathrm{CHCl}_{3}: \mathrm{MeOH}(4: 1)$ foram submetidas à permeação em Sephadex $\mathrm{LH}-20$ e eluídas com $\mathrm{CHCl}_{3}: \mathrm{MeOH}$ (3:7). Desta maneira obteve-se 53,0 mg da luteolina-7-rutenosídeo (4).

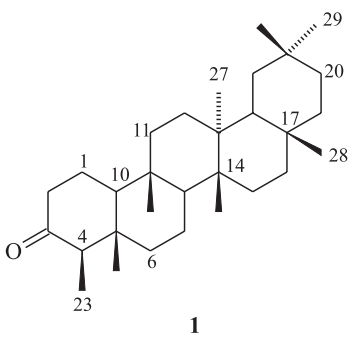

1

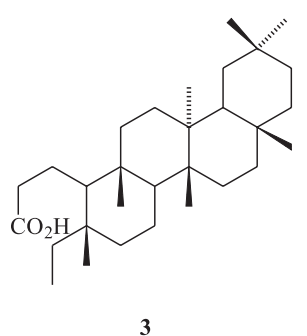

3

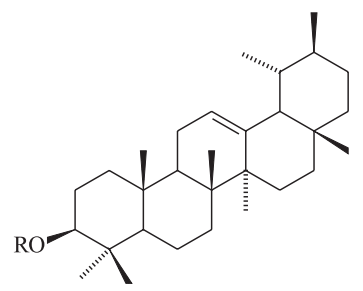

2a $\mathrm{R}=\mathrm{CH}_{3}\left(\mathrm{CH}_{2}\right){ }_{14} \mathrm{CO}$ 2b R $=\mathrm{CH}_{3}\left(\mathrm{CH}_{2}\right)_{16} \mathrm{CO}$

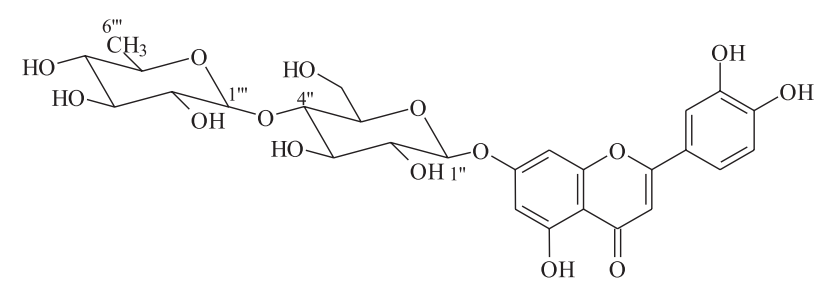

4

Luteolina-7-rutenosídeo (4)

Sólido amorfo. PF $250{ }^{\circ} \mathrm{C}$ (decp.). UV: Tabela 2. Dados de RMN de ${ }^{1} \mathrm{H}[300 \mathrm{MHz}, \mathrm{DMSO}, \delta(\mathrm{ppm}), \mathrm{J}(\mathrm{Hz})]:$ 6,61 ( $\left.s, \mathrm{H}-3\right), 6,04(d$, 1,3 Hz, H-6), 6,38 (d, 1,3 Hz, H-8), 6,38 (d, 2,0 Hz, H-2'), 6,89 (dd, 8,5 e $2,0 \mathrm{~Hz}, \mathrm{H}-5$ '), 7,47 (d, 8,5 Hz, H-6'). RMN de ${ }^{13} \mathrm{C}[75 \mathrm{MHz}$, DMSO, $\delta$ (ppm)]: 167,37 (C-2), 102,90 (C-3), 181,66 (C-4), 161,58 (C-5), 99,43 (C-6), 163,69 (C-7), 95,00 (C-8), 157,88 (C-9), 103,01 (C-10), 120,90 (C-1'), 114, 85 (C-2'), 146,01 (C-3'), 153,02 (C-4'), 117,25 (C-5'), 122,08 (C-6'), valores para o grupo diglicosídeo vide Tabela 1.

\section{Ácido 3,4-seco-friedelan-3-óico (3)}

Cristais brancos. $\mathrm{PF}=216-218{ }^{\circ} \mathrm{C}$. EMIC $\left(\mathrm{CH}_{4}\right) \mathrm{m} / \mathrm{z}$ (int.): 445 $\left[\mathrm{M}^{+*}+\mathrm{H}, \mathrm{C}_{30} \mathrm{H}_{52} \mathrm{O}_{2}\right](21), 443$ (40), 442 (35), 441 (81), 440 (42), 427 (100), 409 (39), 407 (22), 399 (19), 219 (17), 205 (25), 159 (22), 143 (31), 129 (49), 117 (58), 115 (52). IV (KBr) cm ${ }^{-1}: 3200,2929$, 2868, 1705, 11465, 1386, 1284, 1216, 919, 756. RMN de ${ }^{1} \mathrm{H}$ (Tabela 3), $\mathrm{RMN}$ de ${ }^{13} \mathrm{C}$ (Tabela 4).

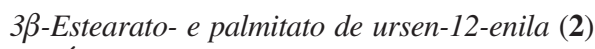

Óleo. RMN de ${ }^{13} \mathrm{C}\left[75 \mathrm{MHz}, \mathrm{CDCl}_{3}, \delta\right.$ (ppm)]: 173,39 $\left(\mathrm{CO}_{2} \mathrm{R}\right)$, 139,52 (C-13), 124,25 (C-12), 80,48 (C-3), 59,10 (C-18), 55,21 (C5), 47,89 (C-9), 42,87 (C-22), 42,75 (C-14), 40,85 (C-8), 38,32 (C-1), 38,01 (C-4), 39,60 (C-19), 39,57 (C-20), 37,01 (C-10), 34,10 (C-17),
31,78 (C-7), 31,43 (C-21), 27,97 (C-15), 28,87 (C-28), 28,60 (C-23), 26,53 (C-16), 23,64 (C-11), 23,54 (C-27), 22,13 (C-2), 21,20 (C-30), 18,11 (C-6), 17,87 (C-29), 16,41 (C-26), 15,87 (C-25), 14,40 (C-24), $13,90\left(\underline{\mathrm{CH}}_{3}\left[\mathrm{CH}_{2}\right]_{\mathrm{n}}\right)$.

Reação de transesterificação

Foram adicionados $20 \mathrm{ml}$ da solução de $\mathrm{NaOMe} / \mathrm{MeOH}(0,5 \mathrm{M})$ a $10 \mathrm{mg}$ da mistura $\mathbf{2 a}+\mathbf{2 b}$. A solução foi mantida sob refluxo durante $2 \mathrm{~h}$, então foi vertido $20 \mathrm{ml}$ de água ao sistema reacional e extraído por duas vezes consecutivas com $40 \mathrm{ml} \mathrm{de} \mathrm{CH}_{2} \mathrm{Cl}_{2}$. A fase diclorometânica foi concentrada obtendo-se a mistura dos ésteres graxos (1,5 mg). EMIE (70 eV): $298\left(\mathrm{M}^{+\bullet}, \mathrm{C}_{19} \mathrm{H}_{38} \mathrm{O}_{2}, 17 \%\right), 270\left(\mathrm{M}^{+\bullet}\right.$, $\left.\mathrm{C}_{17} \mathrm{H}_{34} \mathrm{O}_{2}, 15 \%\right)$.

\section{RESULTADOS E DISCUSSÃO}

O flavonóide foi identificado como sendo a luteolina-7rutenosídeo (4) através da análise dos dados de RMN e pelo deslocamento provocado nas bandas do espectro no ultravioleta, após adição de reagentes de deslocamento. O padrão de substituição do anel A e a atribuição dos deslocamentos químicos foram obtidos a partir da análise dos espectros de RMN de ${ }^{1} \mathrm{H}$ e do HOMOCOSY que permitiram identificar a aglicona como sendo a luteonina. A comparação dos dados de RMN de ${ }^{13} \mathrm{C}$ desta substância com os descritos na literatura ${ }^{6}$ corroboraram a identificação da luteolina-7-rutenosídeo. A confirmação da identificação do diglicosídeo rutinosídeo [raminose $(1 \rightarrow 6)$ glicose] foi efetuada através da análise do deslocamento químico do C-6" no espectro de RMN de ${ }^{13} \mathrm{C}$. No rutenosídeo o sinal do C-6" da glicose é registrado em campo mais baixo $(\sim 6$ $\mathrm{ppm})^{7}$ em relação ao mesmo carbono do monoglicosídeo (Tabela 1). A localização da unidade do açúcar no C-7 foi obtida pela análise dos deslocamentos batocrômicos observados nos espectros no UV. $\mathrm{O}$ deslocamento observado após adição de $\mathrm{AlCl}_{3}$ e, posteriormente, $\mathrm{HCl}$ demonstraram claramente que as hidroxilas HO-5, HO-4' e HO3' estavam livres (Tabela 2).

Tabela 1. Comparação dos dados de $\mathrm{RMN}$ de ${ }^{13} \mathrm{C}$ do rutenosídeo com glicosídeo e raminosídeo

\begin{tabular}{lccc}
\hline C & Rutenosídeo & A-7-O-Glc & A-7-O-Rha \\
\hline $1 "$ & 100,85 & 100,2 & - \\
$2^{\prime \prime}$ & 72,47 & 73,3 & - \\
$3^{\prime \prime}$ & $77,51^{*}$ & 76,6 & - \\
$4^{\prime \prime}$ & $70,85^{*}$ & 69,8 & - \\
$5^{\prime \prime}$ & $77,63^{*}$ & 77,4 & - \\
$6 "$ & 68,92 & 60,9 & - \\
$1^{\prime \prime}$ & 99,27 & - & 98,9 \\
$2^{\prime \prime}$ & $70,79 *$ & - & 70,4 \\
$3^{\prime \prime}$ & $70,22^{*}$ & - & 70,0 \\
$4^{\prime \prime}$ & 72,47 & - & 71,8 \\
$5^{\prime \prime}$ & 68,92 & - & 69,8 \\
$6 "$ & 18,23 & - & 17,7 \\
\hline
\end{tabular}

*Valores podem estar trocados; $\mathrm{A}=$ Apigenina

Tabela 2. Efeitos batocrômicos observados no espectro no ultravioleta de 7 com adição de reagentes $[\lambda(\mathrm{nm})]$

\begin{tabular}{lcc}
\hline Solvente & Banda I & Banda II \\
\hline $\mathrm{MeOH}$ & 350 & 268 \\
$\mathrm{MeOH}+\mathrm{NaOMe}$ & 405 & 265 \\
$\mathrm{MeOH}+\mathrm{AlCl}_{3}$ & 415 & 268 \\
$\mathrm{MeOH}+\mathrm{AlCl}_{3}+\mathrm{HCl}$ & 370 & - \\
$\mathrm{MeOH}+\mathrm{AcONa}^{\mathrm{AcONa}}$ & 400 & - \\
\hline
\end{tabular}


Tabela 3. Correlações H-C (HMQC e HMBC) observadas para o ácido 3,4-seco-friedelan-3-óico (3)

\begin{tabular}{llll}
\hline $\mathrm{H}$ & $\delta(\mathrm{ppm})$ & $\mathrm{C}$ correlacionado $(\mathrm{HMQC})$ & Longa distância (HMBC) \\
\hline 23 & 0,79 & 7,51 & $38,97(\mathrm{C}-5)$ \\
25 & 0,87 & 17,84 & $35,95(\mathrm{C}-11) ; 37,74(\mathrm{C}-9) ; 52,91(\mathrm{C}-8) ; 59,71(\mathrm{C}-10)$ \\
29 & 0,94 & 34,86 & $28,05(\mathrm{C}-20) ; 32,78(\mathrm{C}-21) ; 35,23(\mathrm{C}-19)$ \\
24 & 0,98 & 19,27 & $35,07(\mathrm{C}-4) ; 38,97(\mathrm{C}-5) ; 39,19(\mathrm{C}-6) ; 59,71(\mathrm{C}-10)$ \\
30 & 0,99 & 31,74 & $28,05(\mathrm{C}-20) ; 32,78(\mathrm{C}-21) ; 35,23(\mathrm{C}-19)$ \\
26 & 1,01 & 18,66 & $30,11(\mathrm{C}-12) ; 39,54(\mathrm{C}-13) ; 38,23(\mathrm{C}-14) ; 42,79(\mathrm{C}-18)$ \\
28 & 1,17 & 32,02 & $29,91(\mathrm{C}-17) ; 36,00(\mathrm{C}-16) ; 42,79(\mathrm{C}-18) ; 39,19(\mathrm{C}-22)$ \\
27 & 1,25 & 20,97 & $32,21(\mathrm{C}-15) ; 38,23(\mathrm{C}-14) ; 39,54(\mathrm{C}-13) ; 52,91(\mathrm{C}-8)$ \\
2 & $2,38 d q$ & 38,83 & \\
7 & $0,81 m$ & 18,01 & \\
\hline
\end{tabular}

A natureza triterpênica dos compostos isolados do extrato hexânico foi evidenciada pela coloração rósea obtida na CCD destes, após revelação utilizando-se o reagente de Lieberman-Buchard. A friedelina e o lupeol foram identificados através da comparação direta dos dados de RMN com dados da literatura ${ }^{8}$. A identificação da mistura dos triterpenos lupenona, $\alpha$ - e $\beta$-amirinona foi efetuada baseada na metodologia descrita para análise de triterpenos em mistura por $\mathrm{RMN}^{9} \mathrm{e}$ os dados de $\mathrm{RMN}$ de ${ }^{13} \mathrm{C}$ são semelhantes aqueles descritos na literatura ${ }^{8}$.

$\mathrm{O}$ espectro de $\mathrm{RMN}$ de ${ }^{13} \mathrm{C}$ da mistura $\mathbf{2 a}+\mathbf{2} \mathbf{b}$ apresentou sinais característicos de triterpenos com esqueleto olean-12-eno ${ }^{8}$. No entanto, apresentou sinais de carbonos carboxílicos de ésteres $(\delta 173,39)$ e outros, que evidenciaram efeito de proteção para o sinal do C-2 $(\Delta \delta 5,7 \mathrm{ppm})$ e de desproteção $(\Delta \delta 2,2 \mathrm{ppm})$ para o C-3, com relação aos respectivos carbonos da $\alpha$-amirina. Estes dados foram indicativos de que estes compostos encontravam-se esterificados com ácidos graxos pelo grupo $\mathrm{OH}$ do $\mathrm{C}-3$. A identificação dos ácidos graxos esterificados com a $\alpha$-amirina foi efetuada via CG/EM a partir dos ésteres metílicos graxos, obtidos da reação de transesterificação da mistura de triterpenos com $\mathrm{MeONa} / \mathrm{MeOH}$. O cromatograma de íons totais dos ésteres metílicos formados mostrou dois picos em diferentes tempos de retenção e, cada um destes originou um espectro de massas cujos íons moleculares $(\mathrm{m} / \mathrm{z}, 298$ e 270) e fragmentos eram correspondentes ao estearato de metila e palmitato de metila, respectivamente. Portanto, a análise conjunta dos dados permitiu propor como contituintes da fração a mistura do estearato e palmitato de $\alpha$ amirina. Triterpenos esterificados com os ácidos graxos ainda não haviam sido descritos no gênero, embora já tenham sido descritos na família ${ }^{3}$. Até recentemente, triterpenos simples como as amirinas esterificadas com ácidos graxos ainda eram de distribuição relativamente restrita. Atualmente, o registro da ocorrência desse tipo de substância tem-se mostrado comum, principalmente nas folhas de diversas espécies do gênero Erythroxylum ${ }^{10}$ (Erythroxylaceae).

A substância 3 foi identificada através da análise conjunta dos dados espectrométricos obtidos. O sinal largo entre $3300-2700 \mathrm{~cm}^{-1}$ e a banda em $1705 \mathrm{~cm}^{-1}$ no espectro no infravermelho indicaram a presença de grupo ácido carboxílico. O íon molecular $\mathrm{m} / z$, 444 observado no EM aliado à presença de 30 sinais de carbonos observados no espectro de RMN de ${ }^{13} \mathrm{C}$ permitiram, juntamente com as informações obtidas pelos experimentos DEPT $\left(135^{\circ}\right.$ e $\left.90^{\circ}\right)$, inferir a fórmula molecular de 3 como sendo $\mathrm{C}_{30} \mathrm{H}_{52} \mathrm{O}_{2}$. A partir da análise do espectro de $\mathrm{RMN}$ de ${ }^{1} \mathrm{H}$ desta substância ficou evidenciada a presença de grupo etila devido ao tripleto observado em $\delta$ 0,79. Ainda pelos espectros de HMQC e HMBC este último sinal mostrava correlação com o carbono metílico em $\delta 7,51$ (C-23) e com C-5 ( $\delta 38,97)$, respectivamente (Tabela 3 ). Estes dados sugeriram a presença do anel A aberto neste triterpeno. Esta sugestão estava de acordo com o índice de deficiência de hidrogênio (IDH=5) obtido a partir da FM de 3. Deste modo, a presença de sinais para 7 outros grupos metílicos (singletos) observados no espectro de RMN de ${ }^{1} \mathrm{H}$, bem como a semelhança da maioria dos dados de $\mathrm{RMN}$ de ${ }^{13} \mathrm{C}$ desta substância com a friedelina, permitiram identificar 3 como sendo ácido 3,4seco-friedelan-3-óico, provável produto da oxidação no C3-C-4 da friedelina. A análise dos dados espectrais de $\mathrm{RMN}$ de ${ }^{13} \mathrm{C}$ do ácido 3,4-seco-friedelan-3-óico (Tabela 4) permitiu reatribuir os valores das metilas C-26 e C-27 da friedelina ${ }^{8}$ uma vez que, pelo espectro HMBC do ácido, foram observadas correlações entre H-26 e os C12, C-13 e C-18, enquanto que os hidrogênios metílicos do C-27 apresentavam correlações com C-8, C-14, C-13 e C-15 (Tabela 3, Figura 1). Destaca-se que este ácido foi primeiramente obtido como

Tabela 4. Dados de RMN de ${ }^{13} \mathrm{C}^{*}$ do ácido 3,4-seco-friedelan-3-óico (3) e da friedelina (1) $\left[75 \mathrm{MHz}, \mathrm{CDCl}_{3}, \delta(\mathrm{ppm})\right]$

\begin{tabular}{lccccc}
\hline $\mathbf{C}$ & $\mathbf{3}$ & $\mathbf{1}$ & $\mathbf{C}$ & $\mathbf{3}$ & $\mathbf{1}$ \\
\hline 1 & 21,00 & 22,25 & 16 & 36,00 & 36,05 \\
2 & 35,10 & 41,48 & 17 & 29,91 & 29,99 \\
3 & 178,20 & 213,32 & 18 & 42,79 & 42,87 \\
4 & 35,07 & 58,23 & 19 & 35,23 & 35,35 \\
5 & 38,97 & 42,09 & 20 & 28,05 & 28,14 \\
6 & 39,19 & 41,33 & 21 & 32,78 & 32,49 \\
7 & 18,01 & 18,24 & 22 & 39,49 & 39,24 \\
8 & 52,91 & 53,12 & 23 & 7,51 & 6,75 \\
9 & 37,74 & 37,47 & 24 & 19,27 & 14,63 \\
10 & 59,73 & 59,54 & 25 & 17,82 & 17,92 \\
11 & 35,95 & 35,65 & 26 & 20,03 & $20,12(18,6)$ \\
12 & 30,11 & 30,50 & 27 & 18,61 & $18,61(20,3)$ \\
13 & 39,54 & 39,71 & 28 & 32,01 & 31,78 \\
14 & 38,23 & 38,22 & 29 & 34,86 & 34,98 \\
15 & 32,21 & 32,10 & 30 & 31,74 & 31,71 \\
\hline
\end{tabular}

$\overline{\text { Valores entre parênteses referem-se a atribuições realizadas }}$

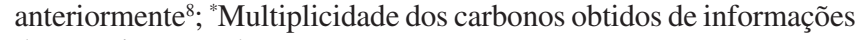
de experimentos de DEPT

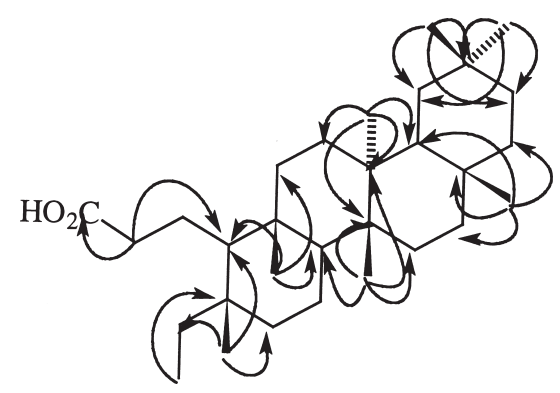

Figura 1. Algumas correlações a longa distância observadas no espectro HMBC do ácido 3,4-seco-friedelan-3-óico (3) 
um dos produtos da irradiação no ultravioleta da friedelina ${ }^{11}$. No entanto, este ácido ainda não havia sido isolado de fonte natural, assim como nem todos os seus dados espectrométricos haviam sido registrados.

\section{AGRADECIMENTOS}

Ao $\mathrm{CNPq}$ pelo apoio financeiro e bolsas de pesquisa. Ao Prof. G. A. Cordell da Universidade de Illinois em Chicago pelos espectros de massas e RMN bidimensionais.

\section{REFERENCIAS}

1. Joly, A. B.; Botânica - Introdução à Taxonomia Vegetal, $4^{\mathrm{a}}$ ed., Companhia Ed. Nacional: São Paulo,1976; Corrêa, M. P.; Dicionário das Plantas Úteis do Brasil e das Exóticas Cultivadas, Instituto Brasileiro de Desenvolvimento Florestal, Ministério da Agricultura: Rio de Janeiro, 1984, vol. III, p. 448.
2. Dias, S. M.; Arq. Inst. Biol. São Paulo 1977, 44, 15.

3. Felício, J. D.; Gonçalez, E.; Lins, A. P.; Braggio, M. N.; David, J. M.; Arq. Inst. Biol. São Paulo 1995, 1/2, 91; Mendes, C. C.; Cruz, F. G.; David, J. M.; Nascimento, I. P.; David, J. P.; Quim. Nova 1999, 22, 185.

4. Aragão, P. C. de A.; Toledo, J. B. de; Moraes, A. A.; Braz-Filho, R.; Quim Nova 1990, 13, 254

5. Davioud, E.; Bailleul, F.; Delaveau, P.; Jacquemin, H.; Planta Med. 1985, 78.

6. Markham, K. R.; Ternai, B. Em The Flavonoids: Advances in Research; Harborne, J. B.; Mabry, T. M., eds.; Chapman and Hall: London, 1986, p. 56; Agrawal, P. K.; Bansal, M. C. Em Carbon-13 NMR of Flavonoids; Agrawal, P.K., ed.; Elsevier: Amsterdam, 1989, p. 325.

7. Markham, K. R.; Ternai, B.; Stanley, R.; Geirger, H.; Mabry, T. J.; Tetrahedron 1978, 34, 1389.

8. Patra, A.; Mukhopadhyay, A. K.; Mitra, A. K.; Org. Magn. Reson. 1981, 17, 166; Mahato, S. B.; Kundu, A. P.; Phytochemistry 1994, 37, 1517.

9. Olea, R. S. G.; Roque, N. F.; Ouim. Nova 1990, 13, 278.

10. Chávez, J. P.; Santos, I. D.; Cruz, F. G.; David, J. M.; Phytochemistry 1996, 41,941 .

11. Kohen, F.; Samson, A.S.; Stevenson, R.; J. Org. Chem. 1969, 34, 1355; Aoyagi, R.; Tsuyuki, T.; Takahashi, T.; Bull. Chem. Soc. Jpn. 1973, 46, 692. 